\title{
Imaging and analysis of forensic striation marks
}

\author{
Michael Heizmann, MEMBER SPIE \\ Universität Karlsruhe \\ Institut für Mess- und Regelungstechnik \\ Postfach 6980 \\ 76128 Karlsruhe, Germany \\ E-mail: heizmann@mrt.uka.de \\ Fernando Puente León, MEMBER SPIE \\ Technische Universität München \\ Lehrstuhl für Messsystem- und \\ Sensortechnik \\ 80290 München, Germany
}

\begin{abstract}
We present a new image processing strategy that enables an automated comparison of striation patterns. A signal model is introduced to describe the interesting features of forensically relevant striation marks. To provide a high image quality, several images of the same surface area are recorded under systematically varying conditions and then combined for an improved result by means of data fusion techniques. Based on the signal model, the signal of interest is concentrated, and a compact representation of the marks is obtained. To enable an efficient description of the relevant features, even in the cases of deformed surfaces or curved striation marks, a straightening of the grooves is performed beforehand. Following, a meaningful "signature" describing the information of interest is extracted using the whole length of the grooves. This signature is used for an objective evaluation of similarity of the striation patterns. (c) 2003 Society of Photo-Optical Instrumentation Engineers. [DOI: 10.1117/1.1622389]
\end{abstract}

Subject terms: automated visual inspection; image processing; data fusion; forensic science; striation patterns; image models; groove straightening

Paper 030164 received Apr. 8, 2003; revised manuscript received May 21, 2003; accepted for publication May 21, 2003.

\section{Introduction}

The analysis and comparison of marks on objects found at a crime scene play an essential role in forensic science. The groove-shaped marks on the cylindrical surface of bullets have been used for a long time to investigate whether two bullets have been fired from the same gun, thus enabling an identification of firearms. In the case of tool marks, which are often found in the context of burglaries and thefts, groove-shaped marks also arise due to the motion of the tool relative to the object touched.

Digitizing and archiving of such marks for subsequent comparisons result in enormous image databases, which may contain some 1,000 marks of the same kind. To establish a correspondence between two marks, an image of the exhibit coming into question has to be compared with the entire database. Up to now, however, this comparison was mainly done visually by experts. Because of the large amounts of comparisons necessary to search the archive, the current matching methods for striation patterns are often unable to provide the turnaround times and success rates that law enforcement demands.

Due to these problems, an automated comparison by means of image processing techniques is desirable. Here, the computer should not primarily serve to visualize the recorded images, but rather to perform a first comparison to assist the expert. The automated inspection comprises the following steps:

- a recording of high-quality images of each mark under reproducible conditions

- a preprocessing to simplify further steps

- the extraction of suitable features

- a feature-level comparison with the entire database.
Stepping up to this challenge, we present a new strategy that enables a fully automated extraction of a meaningful feature set we call a "signature" from an image. Considering the nature of the marks of interest, every processing step meets the specific requirements of groove-shaped textures. Based on the signatures obtained, an objective and efficient comparison of striation patterns can be achieved. ${ }^{1,2}$ Since existing feature extraction algorithms lack a sufficiently high accuracy to permit a reliable processing of arbitrary striation marks, we had to develop a new approach to accomplish this task.

Our strategy is based on the signal model introduced in Sec. 2. Taking the signal properties into account, powerful imaging and preprocessing strategies are presented that yield high-quality images of the marks (see Secs. 3 and 4). Thereafter, the proposed signal model is used to robustly extract the signature of the tool mark, as shown in Sec. 5.

\section{Signal Model}

Neither bullets nor tool marks show a random distribution of groove-shaped marks. In fact, such textures comply with definite rules that are implicitly met at the time of the generation of the grooves. Individual features of the tool edge or the gun barrel emboss the groove structures by their motion relative to the surface that bears the mark. Consequently, all fluctuations of the image intensities along the grooves are bound to represent disturbances that can efficiently be suppressed by performing an averaging in the direction of the grooves. At the same time, such a concentration of the information of interest leads to a considerable data reduction, which contributes to an efficient implementation of the later comparison algorithms. Regarding the classification of grooves, one has to distinguish between marks on pristine bullets on one hand, and marks on deformed bullets as well as tool marks on the other hand. 


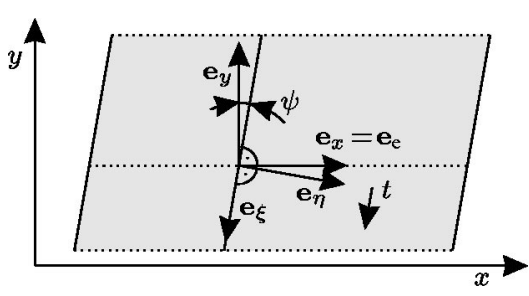

Fig. 1 Signal model for grooves on bullets.

\subsection{Pristine Bullets}

At the time of firing, the kinematics provides for a generation of straight grooves on the cylindrical surface of the bullet. All individual characteristics of the barrel are mapped onto strictly parallel grooves. Due to the twist of the bullet compared to the direction of flight $\mathbf{e}_{y}$, the direction $\mathbf{e}_{\xi}$ of the grooves is tilted by the angle $\psi$ (see Fig. 1). Moreover, the direction of a virtual "tool edge" $\mathbf{e}_{\mathrm{e}}$ describing all of the characteristics of the barrel coincides with the global direction $\mathbf{e}_{x}$.

However, curved grooves may also be found on firearm bullets. The assumption of straight grooves is not always reasonable, especially with bullets that have been heavily deformed due to the impact. In such cases, more complex signal models are needed, such as those used to describe tool marks.

\subsection{Deformed Bullets and Tool Marks}

When dealing with tool marks or deformed bullets, a rectilinear model of the grooves is no longer applicable. The generation process of such marks reveals that the 2-D surface structure results from a combined translation and rotation of a 1-D structure $g(s)$, which in the case of tool marks corresponds with the tool edge. (Although tool edges actually have a spatial character, it is usually sufficient to assume a 1-D edge due to the small extent of most tools perpendicular to the edge direction.) In the case of deformed bullets, the motion inside the gun barrel causes a projection of the individual features on a virtual "tool edge" that embosses strictly straight grooves on the bullet. Here, the curvature of the grooves stems rather from the later deformation of the entire bullet than from the groove generation process.

For all of these reasons, the application of a signal model based on a 1-D, spatially constant profile requires some assumptions.

- First of all, regarding the acquisition of images $d(\mathbf{x})$, where $\mathbf{x}=(x, y)^{T}$ denotes the spatial coordinates, the illumination and the direction of observation have to be kept constant with respect to the groove direction. Consequently, to record images of different marks, all the illumination and positioning parameters should be adjusted exactly and reproducibly. ${ }^{1}$

- Second, a sufficiently large area of the marks should be analyzed to permit a robust estimation of the parameters of the model and to take advantage of its capability of suppressing disturbances.

- It should be assured that no overlaps of the grooves occur, i.e., the tool edge should touch each particular location of the surface only once. With respect to the shape of the grooves, this condition requires continuous paths for all grooves.

- The path of the tool should feature a continuous first derivative. Although this condition may seem to be too restrictive, due to the mass of the tool, mostly differentiable tool paths can be expected.

- Finally, the groove profile has to comply with the requirement of local stationarity. The edge profile is only allowed to vary slowly along the grooves. Such an alteration of the edge profile occurs, for example, when the clearance angle of the tool is changed during the generation of the striae. A reasonable procedure to deal with striation marks showing profile changes would be to split up the entire structure into several sections and to assume a constant groove profile within each section.

Various signal models that lead to such groove structures are conceivable. Some examples are depicted in Fig. 2. Each signal model uses as model parameters the curve $\mathbf{m}(t)$, with the curve parameter $t$ of an arbitrary position on the edge as well as the width of the edge $w$. The parameter $t$ may be, but does not need to be, identical to the running length. Besides, specific model parameters may be necessary, e.g., the center position and the radius of curvature $r$ in the case of circular grooves [see Fig. 2(c)]. Depending on the signal model, correspondences of the local coordinate system $\left(\mathbf{e}_{\xi}, \mathbf{e}_{\eta}\right)$ in the groove direction with the local edge direction $\mathbf{e}_{e}$ or the global coordinate system $\left(\mathbf{e}_{x}, \mathbf{e}_{y}\right)$ are obtained.

With the signal models introduced, the gray-level function $g^{\mathrm{II}}(\mathbf{x})$ of a striation pattern within an image $d(\mathbf{x})$ may be described as follows:

$g^{\mathrm{II}}(\mathbf{x}):=g\left(\{\mathbf{x}-\mathbf{m}[t(\mathbf{x})]\}^{T} \cdot \mathbf{e}_{\mathrm{e}}\right) \cdot \operatorname{rect}\left(\frac{\{\mathbf{x}-\mathbf{m}[t(\mathbf{x})]\}^{T} \cdot \mathbf{e}_{\mathrm{e}}}{w}\right)$,

where $g(s)$ denotes the groove profile in the direction $\mathbf{e}_{e}$ of the edge, and

$\operatorname{rect}(x):=\left\{\begin{array}{ll}1 & \text { for } x \in\left[-\frac{1}{2} ; \frac{1}{2}\right] \\ 0 & \text { otherwise }\end{array}\right.$.

In some cases, Eq. (1) may be simplified. In particular, for circular grooves $\mathbf{e}_{\eta}=\mathbf{e}_{e}$ holds, if the origin is chosen to coincide with the center of the circle [see Fig. 2(c)]:

$\mathbf{m}(\mathbf{x})=r \cdot \mathbf{e}_{\eta}(\mathbf{x})$.

With the Euclidean norm $\|\ldots\|$, the following simplification is obtained:

$g^{\mathrm{II}}(\mathbf{x}):=g\left(\mathbf{x}^{\mathrm{T}} \cdot \mathbf{e}_{\eta}-r\right) \cdot \operatorname{rect} \frac{\|\mathbf{x}\|-r}{w}$.

Such simplifications can be stated for the first four signal models of Fig. 2. However, the resulting equations cannot be transferred to any other signal model. Therefore, an in- 


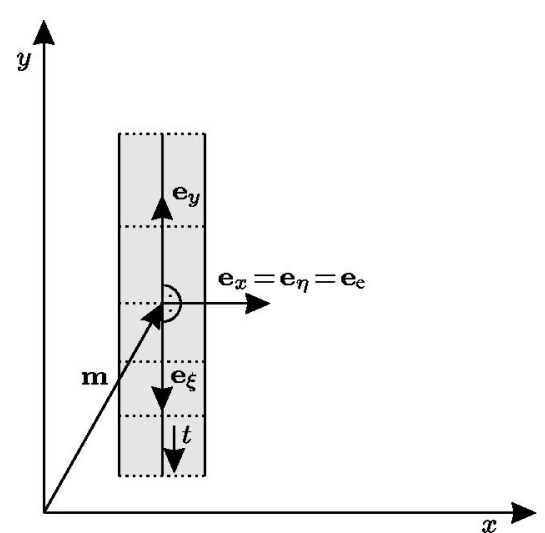

(a)

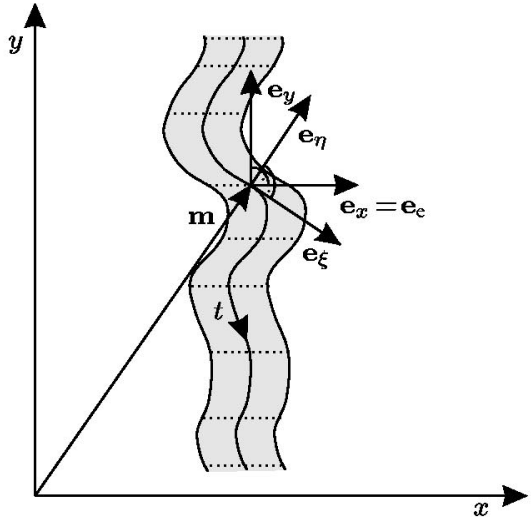

(b)

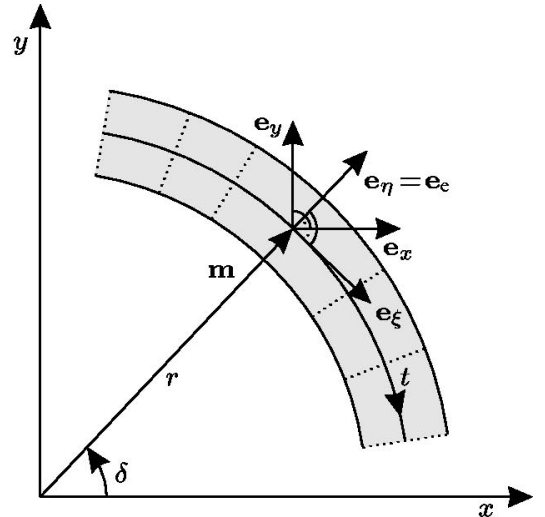

(c)

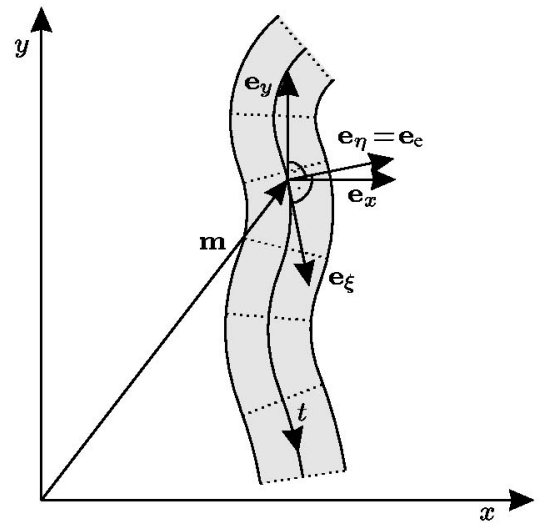

(d)

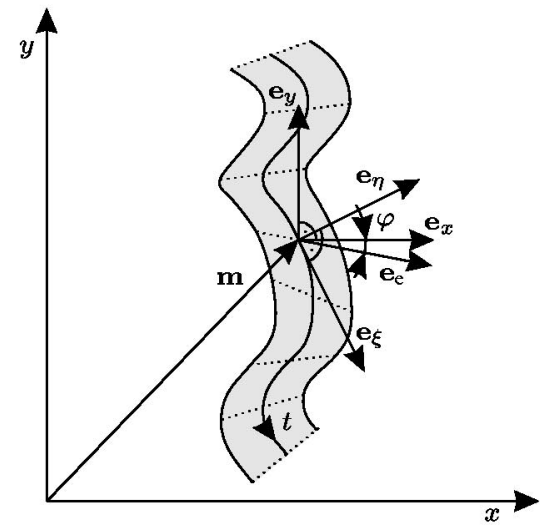

(e)

Fig. 2 Model mechanisms for the generation of grooves: (a) straight grooves; (b) pure translation of tool edge; (c) circular grooves; (d) piecewise circular grooves; and (e) arbitrary translation and rotation of tool edge.

dividual method for the recognition of the course of the grooves has to be developed explicitly for each signal model.

The most general model is the one in which arbitrary translations and rotations of the tool edge are permitted [see Fig. 2(e)]. The only assumption used in this model is that the striae are generated by a 1-D, solid edge. If one was able to reconstruct the path $\mathbf{m}(t)$ of a particular point on the edge as well as the corresponding angle $\angle\left(\mathbf{e}_{x}, \mathbf{e}_{e}\right)$ from the recorded image, then every image point within the groove pattern could be assigned to a particular point on the edge. To some extent, this method would constitute the inversion of Eq. (1).

\section{Imaging}

In forensic science, the requirements concerning the quality of the images to be evaluated are especially high. To achieve a reliable comparison of forensic marks, a reproducible imaging of even the finest marks is necessary. Therefore, great strides must be made in the area of image capture. Ideally, the requirements to be fulfilled by the image acquisition system comprise:

- a high-quality image acquisition providing for high contrast, high resolution, high image sharpness, and high signal-to-noise ratio (SNR) in the whole image
- a thorough coverage of the surface containing the marks of interest

- an easily reproducible recording situation

- a mostly automated image acquisition.

Due to the limitations of optical systems, illumination problems as well as difficulties in imaging all interesting areas simultaneously and with the required resolution, it is often not possible to meet all these requirements with only one image $d(\mathbf{x})$ of the object [see Figs. 3(a) and 3(b)]. Instead, these requirements can be fulfilled by acquiring several images under different recording conditions. If attention is paid to a proper choice of the degrees of freedom of the image acquisition system, and its parameters are varied systematically, the images obtained can then be combined to an enhanced result $r(\mathbf{x})$ matching all requirements, on which the further processing can be based. An example of such an image is shown in Fig. 3(c).

A strategy to generate suitable images $r(\mathbf{x})$ is based on recording a series of images

$\mathcal{D}:=\left\{d\left(\mathbf{x}, \boldsymbol{\omega}_{i}\right), \quad i=0, \ldots, B-1\right\}$,

in which the object distance, the illumination direction, and 


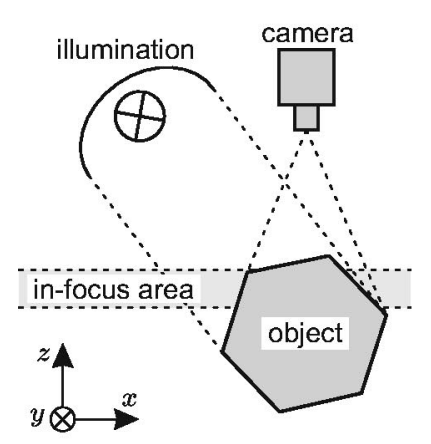

(a)

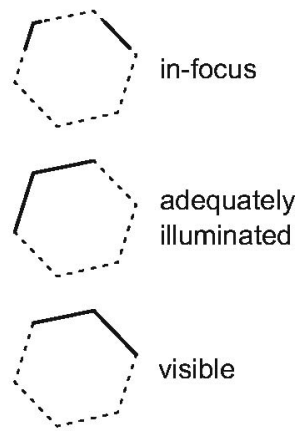

Fig. 3 Image acquisition problems: (a) general scheme; (b) example of an inhomogeneous image of a tool mark according to model Fig. 2(c) obtained with directional lighting; and (c) enhanced image generated by means of fusion techniques.

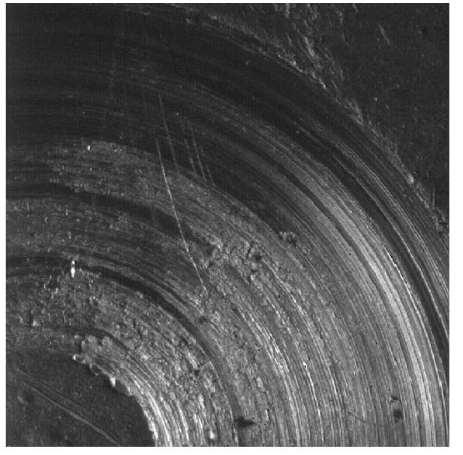

(b)

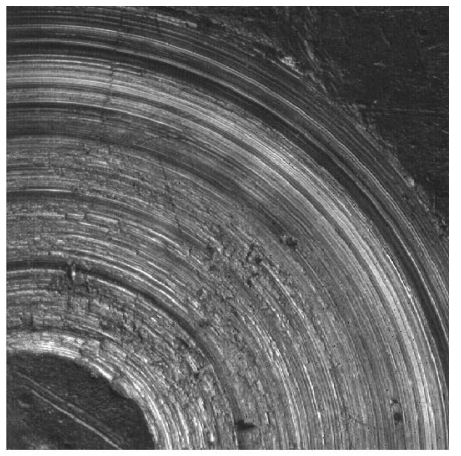

(c) the object pose are all varied, and performing a subsequent multidimensional fusion of the images. ${ }^{3,4}$ The image series is characterized by a parameter vector

$\boldsymbol{\omega}:=\left(\phi, \theta, \zeta, \boldsymbol{\alpha}^{T}, \ldots\right)^{T}$,

describing the recording situation, where $\phi$ and $\theta$ represent the azimuth and the elevation angle of a directional light source, respectively, $\zeta$ denotes the object distance, and $\boldsymbol{\alpha}^{T}$ the object pose with respect to the imaging system. Of course, additional parameters-such as the integration time of the camera or the wavelength of the light source-could be varied too, if necessary. For more information on the impact of image acquisition problems as well as on strategies to compensate them, we refer to Refs. 1,5 , and 6 . In some particular cases, more than a single resulting image will be necessary to describe the marks of interest in their entirety. However, such cases concern constellations of marks showing a complex geometry, and they will not be considered in the scope of this work.

\subsection{Image Capture}

For image striation patterns with high contrast, a suitable illumination has to be chosen. It can be shown that the image intensities obtained from such surfaces highly depend on the direction of the light source, if directional lighting is used [see Fig. 3(b)]..$^{5}$ Since a diffuse illumination pattern can be thought of as a superposition of many single directional light sources from different directions, diffuse lighting will generally lead to a contrast attenuation, and thus to suboptimal results. However, although directional lighting is preferable to illuminate surface structures, the position of the light source has to be adjusted for each location $\mathbf{x}$ of the surface, if an optimal local contrast is wanted.

An important question when recording an image series deals with the strategy for sampling the parameter space $\boldsymbol{\omega}$ with as few images as possible such that 1 . every surface location $\mathbf{x}$ is imaged with high quality in at least one image of the series, and 2. fusion to an improved result $r(\mathbf{x})$ - such as the example shown in Fig. 3(c) - is possible. This problem greatly depends on the object geometry as well as on the surface texture and cannot be dealt with in detail here. However, the following cases of forensically relevant striation marks are treated in more detail.

- Cylindrical surface, straight grooves: To record an image series of cylindrical surfaces showing a single band of straight, parallel grooves, like, e.g., pristine bullets, it is not necessary to vary the illumination space two-dimensionally, because such surfaces only show a high contrast if illumination is perpendicular to the grooves. ${ }^{5}$ Thus, only the elevation angle $\theta$ has to be varied. If the interesting surface areas are not all in-focus simultaneously, the object distance $\zeta$ should be varied, too.

- Planar surface, curved grooves: In the case of planar surfaces containing curved grooves, it is usually sufficient to record an image series in which the azimuth $\phi$ is varied. This case is typical for a wide range of marks generated by screwdrivers and other tools.

- Curved surface, curved grooves: In the most general case of curved surfaces showing curved grooves, such as deformed bullets, both the elevation angle $\theta$ and the azimuth $\phi$ of the light source have to be varied to assure a high quality in at least one image of the series. Additionally, it may be necessary to vary the object distance $\zeta$ and the object pose $\boldsymbol{\alpha}^{T}$ as well to achieve proper focusing and low distortions.

\subsection{Fusion Strategy}

After an image series $\mathcal{D}$ has been acquired, a suitable strategy has to be applied to combine the information of interest distributed over the series to an enhanced result $r(\mathbf{x})$ showing a high quality overall. Such a result is not only advantageous to enable a computerized comparison, but it can also be used to support forensic examiners in matching striae, because a larger area of the surface can be visualized with high contrast simultaneously than when using conventional tools like comparison macroscopes.

In this work, only 1-D illumination series-i.e., series in which only one illumination parameter is varied-are discussed. However, by applying this method in several stages, multidimensional series of images can also be fused. ${ }^{5}$ Figure 4 shows the structure of the fusion algorithm for the case of a varying azimuth $\phi$. 


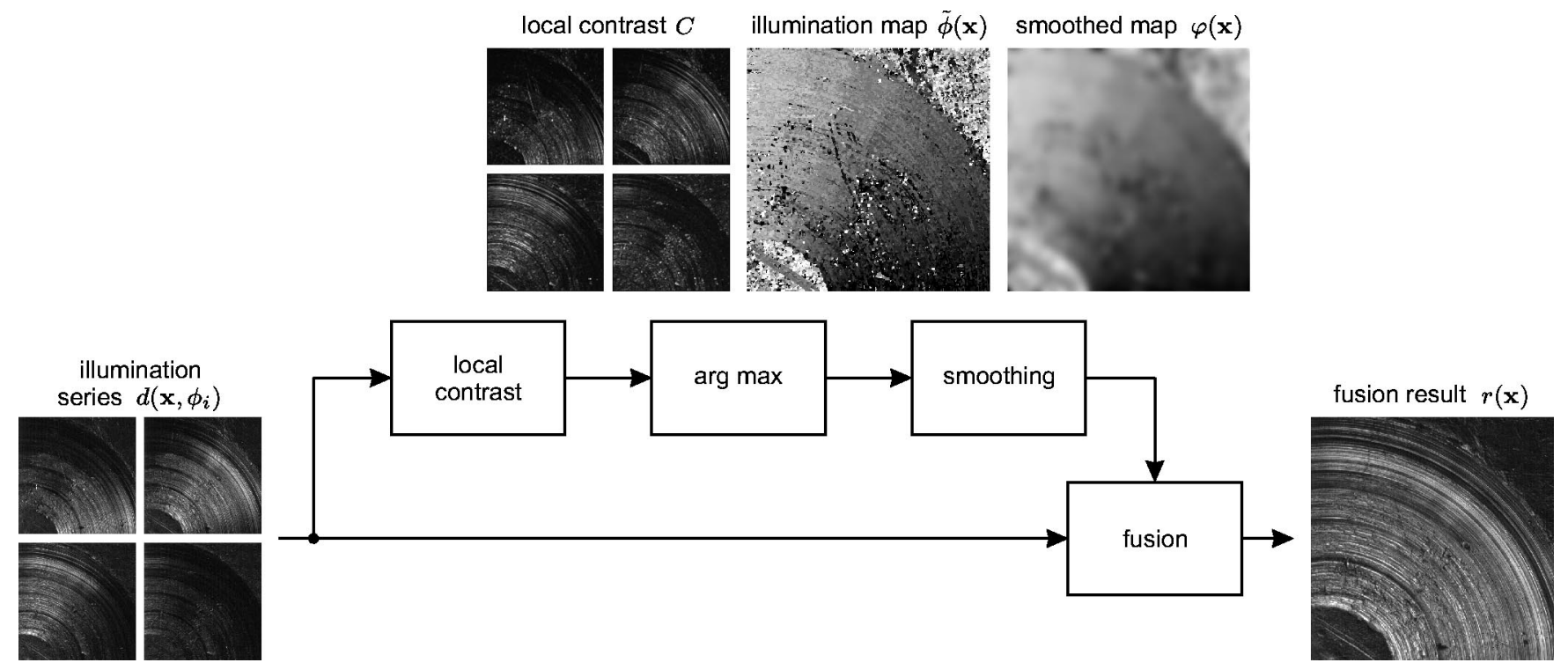

Fig. 4 Structure of the algorithm to fuse 1-D illumination series.

The fusion algorithm is based on the selection of the best illuminated image segments of the series for each location $\mathbf{x}$ based on the maximization of a local criterion $C$. Since a high contrast is desired in our case, the local graylevel variance and the local entropy are suitable critera $C$. The selected illumination direction maximizing the contrast, which is stored for each location $\mathbf{x}$ in the so-called illumination map,

$\widetilde{\phi}(\mathbf{x}):=\underset{\phi_{i}}{\arg \max _{i}} C\left\{d\left(\mathbf{x}, \phi_{i}\right)\right\}$,

has to be a spatial function varying slowly, compared with the signal of interest, i.e., the width of the grooves. This is necessary to avoid artifacts in the fusion result. To assure that this condition is satisfied, a smoothing of the illumination map with a binomial low-pass filter $\operatorname{LP}\{$.$\} is$ performed $^{7}$ :

$\varphi(\mathbf{x}):=\angle \operatorname{LP}\{\exp (\mathrm{j} \widetilde{\phi}(\mathbf{x}))\}$

In this step, the cyclicity of $\phi$ has to be taken into account, because the equation $\phi=\phi+2 \pi k, k \in \mathbb{Z}$, holds. Consequently, not $\widetilde{\phi}(\mathbf{x})$ itself is to be smoothed, but the complex pointer $\exp [\mathrm{j} \widetilde{\phi}(\mathbf{x})]$. The resulting function $\varphi(\mathbf{x})$, which denotes the best-suited local illumination direction, is the angle of the complex result. ${ }^{8}$

The actual fusion is performed by a weighted superposition of two adjacent images $d\left(\mathbf{x}, \phi_{i}\right)$ by means of a linear interpolator $\gamma$, taking the best local illumination direction $\varphi(\mathbf{x})$ into account:

$$
\begin{aligned}
r(\mathbf{x}):= & \frac{\left[\phi_{l}-\varphi(\mathbf{x})\right] \bmod 2 \pi}{\left(\phi_{l}-\phi_{k}\right) \bmod 2 \pi} d\left(\mathbf{x}, \phi_{k}\right) \\
& +\frac{\left(\varphi(\mathbf{x})-\phi_{k}\right) \bmod 2 \pi}{\left(\phi_{l}-\phi_{k}\right) \bmod 2 \pi} d\left(\mathbf{x}, \phi_{l}\right),
\end{aligned}
$$

with

$l:=(k+1) \bmod B$,

$\left(\phi_{l}-\phi_{k}\right) \bmod 2 \pi \leqslant\left[\varphi(\mathbf{x})-\phi_{k}\right] \bmod 2 \pi$,

where $B$ denotes the number of images of the series. The interpolation takes care of a smooth transition between $\phi$-neighboring images. The narrow extent of $\gamma$ ensures an averaging of only similarly illuminated images. Thus, an undesirable contrast loss due to destructive interferences of light and shadow in different images of the series is avoided.

Three properties of the proposed fusion method are responsible for its good performance: 1 . the fusion result $r(\mathbf{x})$ resembles locally the best illuminated image $d\left(\mathbf{x}, \phi_{i}\right)$ of the series; 2 . the smoothness of the selected illumination direction $\varphi(\mathbf{x})$ guarantees that no artifacts are generated in the result $r(\mathbf{x})$; and 3. $r(\mathbf{x})$ achieves globally good results in the sense of maximizing the local contrast $C$.

A fusion of an image series in which the elevation angle $\theta$ of the illumination direction is varied can be performed in an analogous manner. However, since the elevation angle $\theta$ is not cyclic, instead of Eq. (9), the following simplified expression is obtained:

$$
\begin{aligned}
r(\mathbf{x}) & :=\sum_{i} d\left(\mathbf{x}, \theta_{i}\right) \gamma\left[\vartheta(\mathbf{x})-\theta_{i}\right] \\
& =\frac{\theta_{l+1}-\vartheta(\mathbf{x})}{\theta_{l+1}-\theta_{l}} d\left(\mathbf{x}, \theta_{l}\right)+\frac{\vartheta(\mathbf{x})-\theta_{l}}{\theta_{l+1}-\theta_{l}} d\left(\mathbf{x}, \theta_{l+1}\right),
\end{aligned}
$$

with

$\vartheta(\mathbf{x}):=\operatorname{LP}\{\widetilde{\theta}(\mathbf{x})\}, \quad \widetilde{\theta}(\mathbf{x}):=\underset{\theta_{i}}{\arg \max _{i}} C\left\{d\left(\mathbf{x}, \theta_{i}\right)\right\}$ 

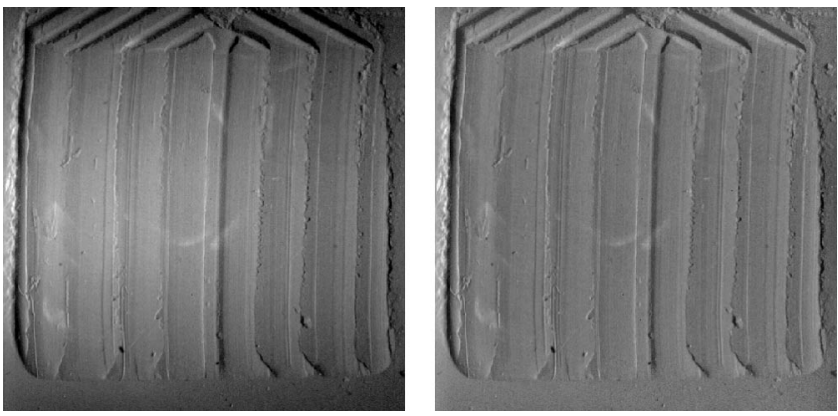

Fig. 5 Example of the preprocessing of a tool mark: (left) original image; (right) homogenization result.

\section{Preprocessing}

After having generated a high-quality image showing the marks of interest by means of the approach presented in Sec. 3.2, a preprocessing is performed to suppress texture inhomogeneities that arise from the illumination process and from the object shape. At the same time, the further processing steps will be simplified. To this end, a directional Gaussian high-pass filter is used that eliminates slowly varying gray-level fluctuations perpendicular to the grooves and provides for a homogenization of first degree (in image processing literature, this step is sometimes referred to as a shading correction), i.e., of the local average gray level, without generating undesirable artifacts. ${ }^{9,10}$ Figure 5 shows an example of the preprocessing.

Alternatively, a homogenization of second degree, in which the local contrast is equalized as well, ${ }^{9}$ has also been tested. However, since this powerful homogenization method leads to a significant alteration of the signal of interest, it appeared to be a less indicated preprocessing tool for quantitative image comparison. ${ }^{1}$

\section{Extraction of a Signature}

To enable an efficient computerized comparison of marks, the database matching should not be performed at the image level. Instead, a data reduction should be accomplished first to extract from the image a signature, i.e., a 1-D "fingerprint" of the striation pattern. The resulting signature can be considered characteristic for a certain specimen of a tool or firearm.

The signal model proposed in Sec. 2 is based on the kinematics imposed during the generation of grooves. It would be ideal-if one was able-to infer the 1-D edge directly from an image of the marks. This would represent the inversion of the kinematics of the groove generation (see Fig. 6). However, such an immediate strategy does not seem to be feasible, since usually the kinematics on the groove generation cannot be determined. Therefore, a method is needed to extract the signature without an exact knowledge of the process that generated the striae.

Many of the strategies applied up to now to extract such signatures are based on sampling the image intensities of the groove structure along a straight line perpendicular to the striations direction. ${ }^{11,12}$ Sometimes, a small amount of profiles specified interactively is averaged. However, a computerized optimization of the parameters determined thereby (e.g., the angle to the local direction of the grooves) is generally not performed.

The main drawback of these strategies is their high susceptibility to all kinds of disturbances. The texture of a striation pattern may appear degraded by a large variety of factors, including scratches, and soiling, but also by cavities and inclusions when castings are used (see, e.g., Fig. 5). For this reason, a robust strategy to trace and straighten the grooves is proposed next. The underlying idea is to consider the whole length of the grooves to suppress the influence of local disturbances on the resulting signature. Since such disturbances only affect minor regions of the marks, a suitable filtering along the grooves can reduce their influence. Filtering the gray values along a groove yields a single point of the signature. By repeating the groove tracing for all grooves of a striation pattern, the desired signature is obtained.

\subsection{Pristine Bullets}

The proposed strategy can easily be illustrated with pristine bullets. As shown in the applicable signal models [Figs. 1 and 2(a)], the angle $\psi$ describes the global direction of the grooves. This angle has to be estimated to perform the projection in the direction of the striae. This can be done, e.g., based on the analysis of a radial projection of the periodogram. ${ }^{1,13}$

\subsection{Deformed Bullets and Tool Marks}

If the local orientation of the grooves changes within the area of interest, it turns out to be more complicated to consider the whole length of the grooves. Here, every image point has to be assigned individually to a particular position on the unidimensional edge. The grooves have to be traced along the whole area of the mark. In this case, suitable strategies to generate a signature include a local orientation analysis and model-based methods.

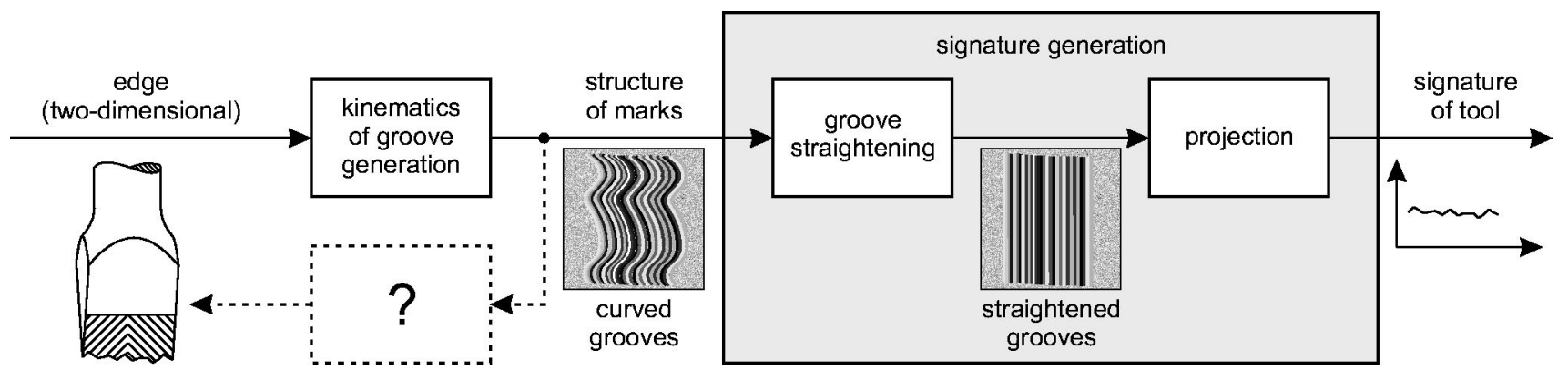

Fig. 6 Groove straightening and generation of a signature. 


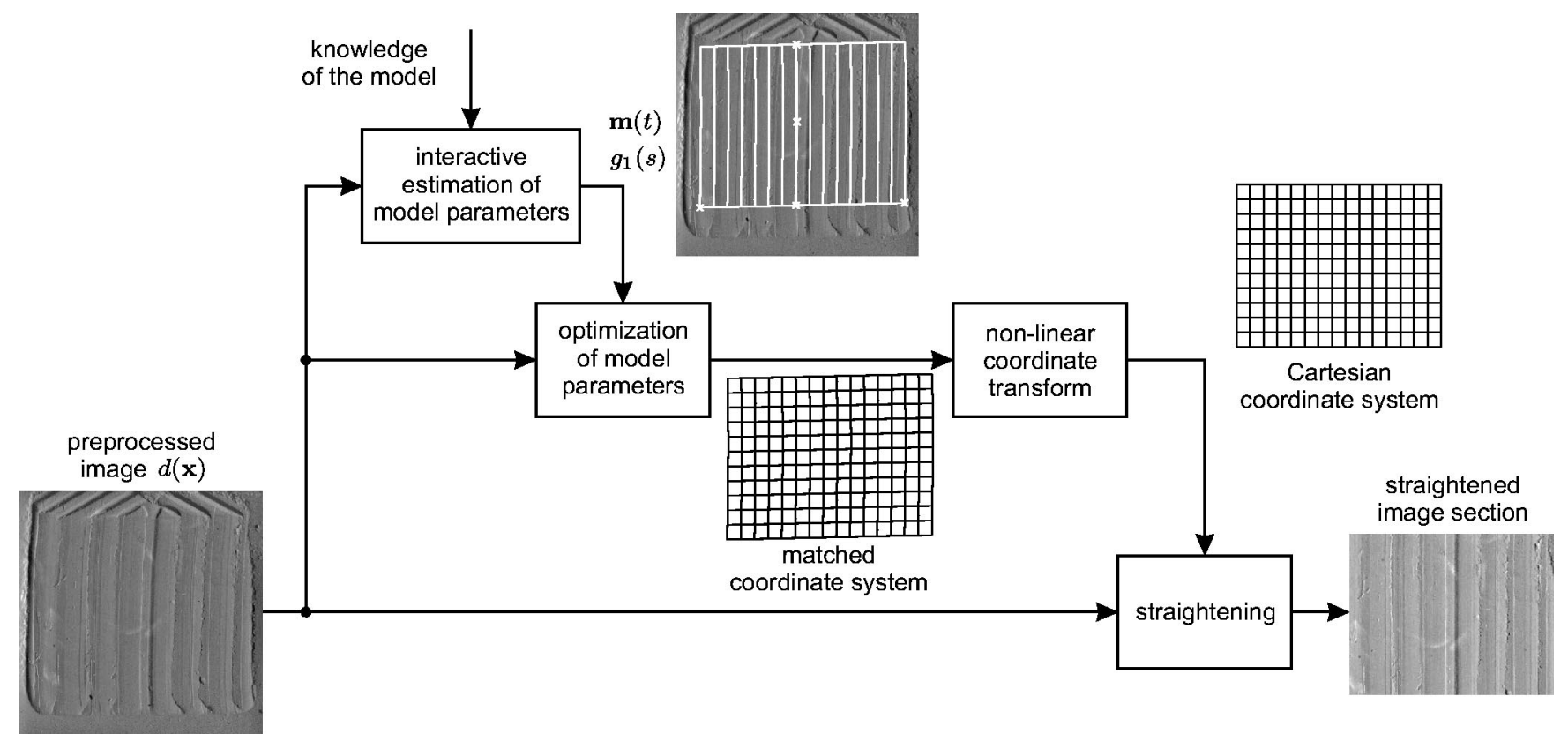

Fig. 7 Model-based groove straightening algorithm.

\subsubsection{Local orientation analysis}

These methods allow us to determine the local direction of a texture (e.g., of a striation pattern) based on local features, such as the variance or gradients of gray values, or a local Fourier analysis. ${ }^{1,14}$ In the context of textures on technical surfaces, local orientation methods have proven to perform satisfactorily. ${ }^{13}$ They have also been successfully applied to the processing of striation marks on deformed bullets. ${ }^{1}$

However, a reliable identification of the local striation direction requires that the directionality of the surface texture is locally sufficiently pronounced. Concerning the processing of tool marks, a segmentation of the texture into regions containing grooves and background regions has to be performed first. Moreover, tool marks often do not show sufficiently pronounced grooves over their whole width. This leads regularly to errors that constrain the usability of this approach considerably.

\subsubsection{Model-based groove tracing}

Model-based strategies according to Sec. 2 allow us to infer the path of the tool edge from the arrangement of the striae in the image. The corresponding groove straightening algorithm is illustrated in Fig. 7, with marks generated by a pure translation of the tool edge. Marks described by other signal models and their corresponding parameters can be treated analogously.

Starting from the preprocessed image $d(\mathbf{x})$, an adequate signal model has to be chosen. The selected model determines the parameters needed. Concerning the example of Fig. 7, the assumption of a pure translation of the tool edge [Fig. 2(b)] seems to be reasonable. One may object that this simplifying assumption holds only for a relatively small class of striation patterns. However, when applied locally, the corresponding model depicted in Fig. 2(b) has proven to yield both satisfactory results and a good computational performance in most practical cases. In this case, an edge line $g_{1}(s)$ describing the direction and the width of the edge as well as a guiding groove $\mathbf{m}(t)$ are required as model parameters. The arguments $s$ and $t$ describe the position on the edge and the curve parameter, respectively.

First of all, both model parameters - edge line and guiding groove-are estimated interactively by the forensic examiner [see Fig. 8(a)]. The edge line is defined as the width of the interesting region of the mark in the direction of the edge (points 0,1$)$. To estimate the guiding groove, several points on a particularly pronounced groove are manually marked (points 2 to 4). To comply with the postulate of smoothness for the marked groove, the points are interpolated by means of splines. However, it should be emphasized that an interactive estimation of the path of the grooves does not seem to be adequate for a few reasons: the precision of the method would suffer in the case of inaccurate user input; and for reasons of reliability and reproducibility, any unsupervised adoption of data determined interactively should be avoided.

To cope with these difficulties, a computer-aided optimization of the path of the guiding groove is performed in which the interactive estimation is used as the initial state. Several approaches are available to accomplish this task. In the following, an algorithm is presented that performs an optimization of the position of all points configuring the discrete guiding groove by maximizing the local crosscorrelation coefficient.

An alternative approach consists in regarding the guiding groove as an active contour or snake. ${ }^{15}$ With some slight adaptation concerning the definition of the internal energy, such a snake can be fitted to the optimal groove path. Furthermore, the application of snake algorithms to the control points of a spline describing the guiding groove constitutes another possibility to enhance the initial estimate of the guiding groove. ${ }^{16}$ The advantage of those ap- 


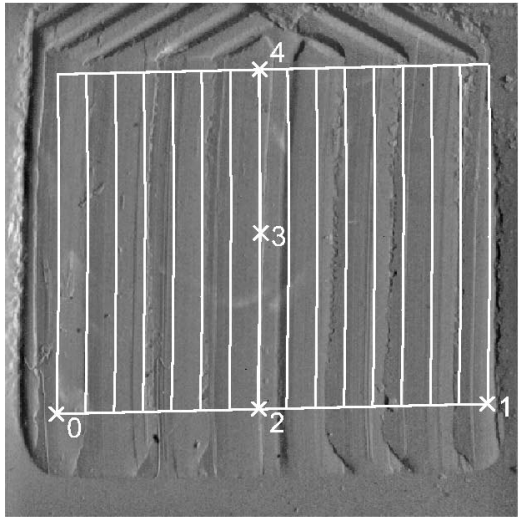

(a)

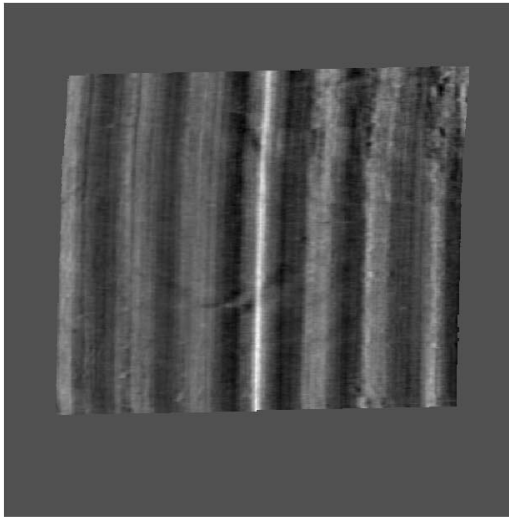

(b)

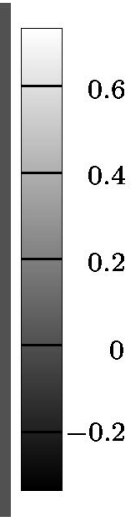

Fig. 8 Groove straightening: (a) interactive estimation of the guiding groove; (b) respective correlation image; and (c) extraction of the signals $g_{1}(s)$ and $g_{2}(s)$. proaches based on snakes consists of the implicit consideration of continuity conditions. On the other hand, these methods entail a significantly increased computational expense.

The cross-correlation function $(\mathrm{CCF})$ is a well-known tool to detect linear correspondences. ${ }^{17}$ In forensic science, its application to matching striation patterns has also proven to provide successful and robust results. ${ }^{1,18}$ The $\mathrm{CCF}$ is defined as

$k_{12}(\tau):=\widetilde{g}_{1}(s) \circledast \widetilde{g}_{2}(s)=\int_{-\infty}^{\infty} \widetilde{g}_{1}(s) \cdot \widetilde{g}_{2}(s-\tau) \mathrm{d} s$,

where both signals $g_{i}(s)$ have been normalized by centering them around their mean values $m_{g i}$ and dividing them by their standard deviations $s_{g i}:=\left(\operatorname{var}\left\{g_{i}(s)\right\}:\right)^{1 / 2}$

$\widetilde{g}_{i}(s):=\frac{g_{i}(s)-m_{g i}}{s_{g i}}$.

The value of the maximum $\rho_{12}:=\max \left\{k_{12}(\tau)\right\}$ indicates the degree of similarity between both signals $g_{1}(s)$ and $g_{2}(s)$. The nearer $\rho_{12}$ lies to the maximal value 1 , the stronger their resemblance will be. The location of the maximum of the $\mathrm{CCF}$

$\tau_{0}:=\arg \max \left\{k_{12}(\tau)\right\}$,

supplies the information on how to choose the shift between both signals to achieve the best possible match.

The signal $g_{1}(s)$ is taken directly from the edge selected interactively and serves as a reference pattern for the following correlation [see Fig. 8(c)]. According to its definition, it is spatially limited and has the length $L_{1}$. It might be reasonable to weight the intensity values around the marked groove, e.g., by means of a Gaussian $G(s, \sigma)$. This way, a higher weight of the central region is obtained as compared with more distant parts. Since defects of the groove pattern are more likely to appear at the border of the mark, their influence on the CCF is thus reduced. The origin of $g_{1}(s)$ is positioned on the guiding groove. For every point $\mathbf{m}(t)$ of the guiding groove, the corresponding signal $g_{2}(s)$ is determined by interpolating the intensities along a

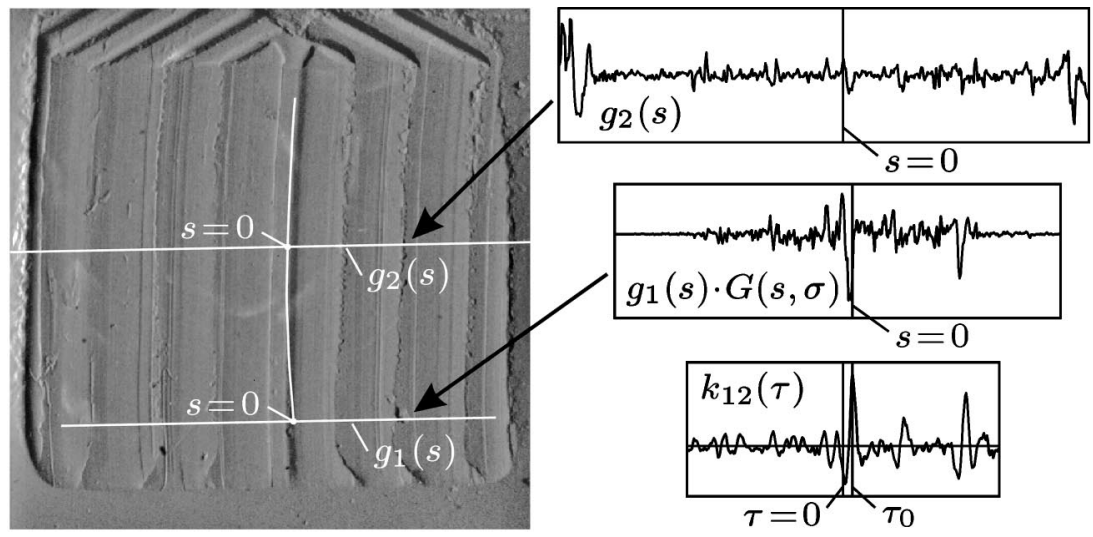

Fig. 9 Direct correlation of two signals parallel to the tool edge extracted from the preprocessed image. 


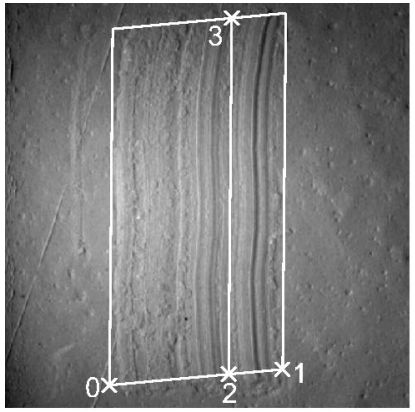

(a)

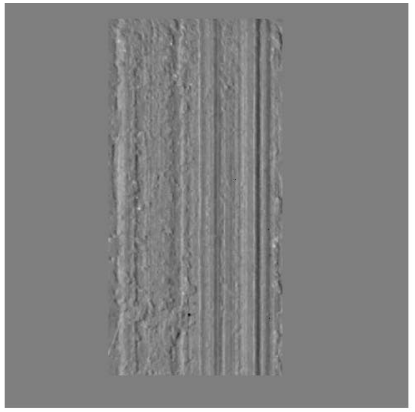

(b)

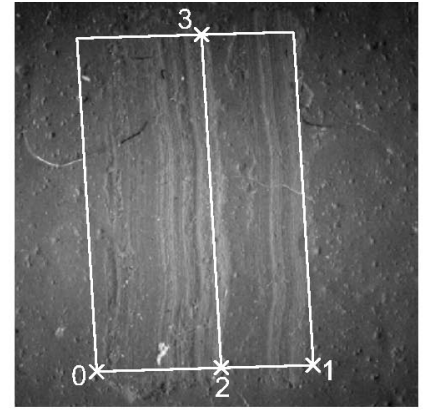

(c)

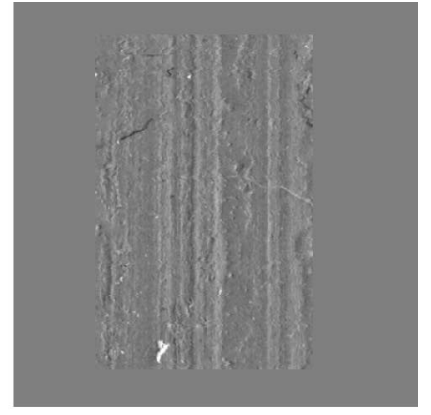

(d)

Fig. 10 Experimental results: (a), (c) original images; (b), (d) preprocessed and straightened image sections.

straight line through $\mathbf{m}(t)$ parallel to the edge line. The origin $g_{2}(s=0)$ is also set on the guiding groove. In contrast to $g_{1}(s)$, the length $L_{2}$ of the signal $g_{2}(s)$ is not limited to a defined value but is restricted by the image boundaries.

The location $\tau_{0}$ of the maximum of the CCF yields the shift leading to the highest similarity between the reference profile $g_{1}(s)$ and the groove profile $g_{2}(s)$. If $\tau_{0}$ differs from zero for a certain point $\mathbf{m}(t)$, an unprecise user input is assumed, and the guiding groove is shifted at that very point by $\tau_{0}$ parallel to the edge line to correct this error.

To give an example, Fig. 9 depicts two signals extracted from a preprocessed image of a tool mark as well as their corresponding $\mathrm{CCF} k_{12}(\tau)$. In this case, a Gaussian was used before to weight the signal $g_{1}(s)$. The CCF shows a strongly pronounced maximum with $\rho_{12}=0.43$. Although this value may seem to be too low for other applications of the CCF, the only important property in our context is a reliable identification of the global maximum. In this respect, the value obtained suffices for a further evaluation.

The CCFs calculated at every position of the guiding groove can be coded as gray values and assembled to a correlation image [see Fig. 8(b)]. The origin of each CCF has been chosen to coincide with the guiding groove, whereas the CCFs themselves have been plotted parallel to the edge line. The maxima $\rho_{12}$ of the CCFs can be recognized as a bright curve in the correlation image. Thus, despite the very inaccurate input of the guiding groove in the example of Fig. 8(a), the correlation image in Fig. 8(b) faithfully describes its actual path. The final result of the proposed optimization strategy is equivalent to a matched coordinate system that can be used to perform a precise straightening of the grooves.

\subsection{Groove Straightening}

The optimized model parameters yield a matched coordinate system in which the groove direction corresponds locally to the ordinate. The abscissa is not affected by the optimization and represents the edge direction. By means of a nonlinear transform of the matched coordinate system into a Cartesian coordinate system, a straightened image is obtained (see Fig. 7). Based on this result, the signature can easily be computed by averaging the image rows.

\section{Experimental Results}

To substantiate a good performance of the signal processing steps proposed in this work, Fig. 10 shows examples of typical tool marks and the results of our preprocessing and straightening strategies. In Figs. 10(a) and 10(c), an overlay with the interactive estimation of the model parameters has been plotted. As stated in Sec. 5.2, a pure translation of the tool edge has been found to be suitable to describe the groove generation in the case of most tool marks.

Even though both original images show considerable distortions, such as an inhomogeneous illumination and, in the case of Fig. 10(c), dust particles, the processing generates satisfactorily straightened grooves that can easily be concentrated into signatures. However, when the groove texture becomes too faint or parts of the grooves are discontinuous, the straightening approach reaches its limits. This can be seen in the upper part of Fig. 10(d), where a sufficient overall straightening still can be achieved, but slight deviations from a strictly straight appearance of the texture have to be tolerated.

\section{Conclusions}

A model-based description of the structure of a wide range of forensically relevant marks, such as tool marks, marks on firearm bullets, and the like, is introduced. Aided by this model, a strategy to generate high-quality images of these marks is proposed. To obtain reliable signatures, a novel methodology to trace and straighten the groove-shaped marks is discussed.

The resulting representation describes the entire tool edge faithfully. Consequently, it is straightforward to use it for a subsequent comparison at a signal level. To this end, approaches based on correlation methods have shown to provide satisfactory results. ${ }^{17}$ Furthermore, the presented strategy can also be combined with existing systems to improve their performance and reliability. Together with suitable comparison approaches, a highly robust and computationally efficient comparison framework is obtained that provides the turnaround times and success rates that a forensic identification of marks demands.

\section{Acknowledgments}

The authors would like to thank Mr. Andreas Kapp and Mr. Thao Dang for valuable comments on a draft version of this work. 


\section{References}

1. F. Puente León, Automatische Identifikation von Schußwaffen, VDI Verlag, Düsseldorf (1999).

2. F. Puente León and J. Beyerer, "Automatic comparison of striation information on firearm bullets," Proc. SPIE 3837, 266-277 (1999).

3. J. J. Clark and A. L. Yuille, Data Fusion for Sensory Information Processing Systems, Kluwer Academic Publishers, Boston (1990).

4. X. E. Gros, NDT Data Fusion, Arnold, London (1997).

5. F. Puente León, "Enhanced imaging by fusion of illumination series," Proc. SPIE 3100, 297-308 (1997).

6. G. H. Tarbox and S. N. Gottschlich, "Planning for complete sensor coverage in inspection," Comput. Vis. Image Underst. 61(1), 84-111 (1995).

7. B. Jähne, Digital Image Processing: Concepts, Algorithms, and Scientific Applications, Springer-Verlag, Berlin (1993).

8. K. V. Mardia, Statistics of Directional Data, Academic Press, London (1972).

9. J. Beyerer and F. Puente León, "Suppression of inhomogeneities in images of textured surfaces,” Opt. Eng. 36(1), 85-93 (1997).

10. T. Lindeberg, Scale-Space Theory in Computer Vision, Kluwer Academic Publishers, Boston (1994).

11. W. Deinet, "Studies of models of striated marks generated by random processes," J. Forensic Sci. 26(1), 35-50 (1981).

12. Z. Geradts, J. Keijzer, and I. Keereweer, "A new approach to automatic comparison of striation marks," J. Forensic Sci. 39(4), 974-980 (1994).

13. J. Beyerer, "Model-based analysis of groove textures with applications to automated inspection of machined surfaces," Measurement 15, 189-199 (1995).

14. M. Kass and A. Witkin, "Analyzing oriented patterns," Comput. Vis. Graph. Image Process. 37(3), 362-365 (1987).

15. M. Kass, A. Witkin, and D. Terzopoulos, "Snakes: active contour models," Int. J. Comput. Vis. 1(4), 321-331 (1987).

16. R. Cipolla, Active Visual Inference of Surface Shape, Springer-Verlag, Berlin (1995).

17. F. Puente León and M. Heizmann, "Strategies to detect non-linear similarities by means of correlation methods," Proc. SPIE 4572, 513524 (2001)

18. M. Heizmann, "Strategies for the automated recognition of marks in forensic science," Proc. SPIE 4709, 68-79 (2002).

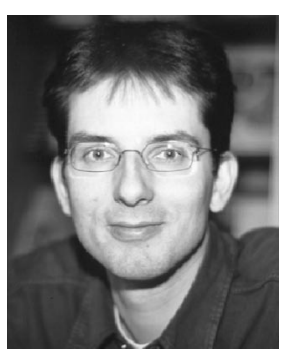

Michael Heizmann received his MS degree in mechanical engineering in 1998 from the University of Karlsruhe, Germany. Since September 1998, he has been a research assistant with the Institut für Messund Regelungstechnik of the University of Karlsruhe. His research interests include image processing, automated visual inspection, and their applications in industry and forensic science.

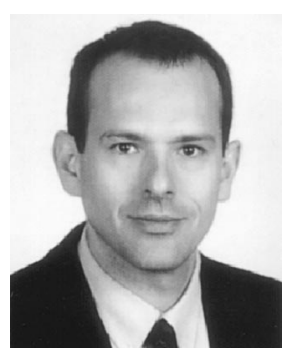

Fernando Puente León is a professor in the Department of Electrical Engineering and Information Technology at the Technische Universität München, Germany. He received his MS degree in electrical engineering in 1994 and his PhD degree in automated visual inspection in 1999, both from the University of Karlsruhe, Germany. From 2001 to 2002, he was employed by Design of Systems on Silicon (DS2), Valencia, Spain. From 2002 to 2003, he was a postdoctoral research associate at the Institut für Mess- und Regelungstechnik of the University of Karlsruhe, where he headed the Information Fusion Group. His research interests include image processing, automated visual inspection, information fusion, measurement technology, and communications. 